Research Article

\title{
Preparation and Characterization of Keratin/Alginate Blend Microparticles
}

\author{
Yaowalak Srisuwan and Prasong Srihanam \\ Creative and Innovation Chemistry Research Unit, The Center of Excellence for Innovation in Chemistry, \\ Department of Chemistry, Faculty of Science, Mahasarakham University, Maha Sarakham 44150, Thailand \\ Correspondence should be addressed to Prasong Srihanam; prasong.s@msu.ac.th
}

Received 16 October 2017; Revised 26 December 2017; Accepted 27 December 2017; Published 20 February 2018

Academic Editor: Renal Backov

Copyright (c) 2018 Yaowalaker Srisuwan and Prasong Srihanam. This is an open access article distributed under the Creative Commons Attribution License, which permits unrestricted use, distribution, and reproduction in any medium, provided the original work is properly cited.

\begin{abstract}
The water-in-oil (W/O) emulsification-diffusion method was used for construction of keratin (Ker), alginate (Alg), and Ker/Alg blend microparticles. The Ker, Alg, and Ker/Alg blend solutions were used as the water phase, while ethyl acetate was used as the oil phase. Firstly, different concentrations of Ker solution was used to find suitable content. 1.6\% w/v Ker solution was blended with the same concentration of the Alg solution for further microparticle construction. Results from scanning electron microscope analysis show that the microparticles have different shapes: spherical, bowl-like, porous, and hollow, with several sizes depending on the blend ratio. FTIR and TG analyses indicated that the secondary structure and thermal stability of the microparticles were influenced by the Ker/Alg blend ratio. The interaction between functional groups of keratin and alginate was the main factor for both $\beta$-sheet structure and $T_{d, \max }$ values of the microparticles. The results suggested that Ker/Alg blend microparticles might be applied in many fields by varying the Ker/Alg ratio.
\end{abstract}

\section{Introduction}

Study on protein materials such as collagen, gelatin, albumin, and silk fibroin and keratin has been increased gradually due to their higher environmental safety than synthetic materials. Keratin is a structural protein derived from various organs including epithelial cells [1], human hair, quills, wool, horns, hooves, and nails of animals, as well as feathers, claws, and beakers of birds and reptiles [2]. The keratin proteins effectively serve a variety of functions, especially for protection and defense. Previous reports have shown that the keratin protein have unique characteristics of bioactivity, biocompatibility, biodegradability, and natural abundance. In recent years, keratin has been applied in wound healing [3], nerve and bone regenerations, hemostasis and cell culture [4], water purification, textile finishing, and composite materials [5]. Therefore, a keratin-based material is a very attractive construction. Keratin-based biomaterials such as films, hydrogels, dressing, and scaffolds have been prepared and practically applied in many fields [6].
Biopolymer-based materials are wildly used according to their user-friendly and eco-friendly features. The large raw materials originate sources of biopolymers are agricultural and marine due to their hydrophilic character, high degradation, as well as simple combined with additives [7]. Alginate (Alg) is a natural polysaccharide extracted from seaweeds [8]. It is an anionic copolymer of $1,4-\beta$-D-mannuronic acid (M blocker) and L-guluronic acid (G blocker) units, which has an irregular pattern of varying proportions of GG, MG, and MM blocks [9]. Alginate which composed of free hydroxyl and carboxyl groups distributed along the backbone is an ideal candidate for chemical modification. In addition, alginate can be formed as a hydrogel when induced by divalent cations such as calcium $\left(\mathrm{Ca}^{2+}\right)$ and barium $\left(\mathrm{Ba}^{2+}\right)$ [10]. Alginate and its derivatives have been widely used in term of biomaterials in various fields including cell immobilization, tissue engineering, drug delivery, controlled release, and food application [11]. This was due to its biological properties such as low immunogenicity and low toxicity and forming stable hydrogel under physiological conditions. The alginate hydrogels have 
been constructed in different forms including micro- and macroscale beads, fibers, films, and 3D gels [12]. Alginate hydrogel is popularly used according to highly permeable to solute transfer and it can be blended with other natural materials such as halloysite $[7,10,13]$ to fabricate new types of hydrogel based biomaterials.

The interest in the particle form of biomaterials has been focused on their potential applications in drug carrier delivery system, cosmetics, dyes and inkers, cell culture, and catalytic and selective materials. Several techniques such as water-in-oil in water emulsion solvent extraction/evaporation, oil-in-water in emulsion core template extraction, oil-in-water in oil emulsion hydration, spray drying, and electrospraying have been used for microparticle preparation of biomaterials [14]. Until now, there are few reports of human hair keratin/alginate blend particle construction. The purpose of this work is to prepare keratin microparticles using the water-in-oil (W/O) emulsification-diffusion concept which was developed by our research unit. The organic solvent, ethyl acetate, was used as an oil phase while the keratin (Ker)/alginate (Alg) aqueous solution was used as a water phase. The effects of W/O volume ratios and Ker or Alg concentrations on prepared microparticles' appearances were investigated and discussed.

\section{Materials and Methods}

2.1. Materials. Human hair was collected from a local hair salon in Maha Sarakham Province, Thailand. The hair was wormed at $40^{\circ} \mathrm{C}$ before immersing in $n$-hexane for $12 \mathrm{~h}$ to remove some lipids and dirties. Hexane, $\mathrm{NaOH}$, SDS, and urea with analytical grade were purchased from UNIVAR, Ajax Finechem, and used without further purification. Sodium alginate was purchased from Thermo Fisher Scientific.

Keratin from human hair was extracted using modification of the Shindai method [15]. Briefly, $10 \mathrm{~g}$ of hair sample was dissolved using mixture solution of $8 \mathrm{M}$ urea, $0.26 \mathrm{M}$ sodium dodecyl sulfate (SDS), and $0.4 \mathrm{M} \mathrm{NaOH}$ in $100 \mathrm{~mL}$ distilled water at $50^{\circ} \mathrm{C}$ with stirrer until homogeneous dissolution was occurred. The obtained solution was then dialyzed in cellulose tubular membranes (molecular weight cutoff $3.5 \mathrm{kDa}$, Thermo Scientific, USA) against distilled water for 3 days. Finally, the Ker solution concentration was adjusted to $2 \% \mathrm{w} / \mathrm{v}$ with distilled water. For blending solution, 2\% (w/v) alginate solution was prepared by dissolving sodium alginate in distilled water.

2.2. Preparation of Microparticles. The Ker microparticles with and without surfactant were prepared by the water-in-oil (W/O) emulsification-diffusion method. Firstly, $100 \mathrm{~mL}$ of ethyl acetate in beaker was kept on the magnetic stirrer, and then an appropriate amount of Ker (different concentrations: $0.4,0.8$, and $1.6 \% \mathrm{w} / \mathrm{v}$ ) solution was added slowly dropwise into the solvent with stirring continuously at $900 \mathrm{rpm}$ for $30 \mathrm{~min}$. During emulsification and diffusion processes, the beaker was covered with an aluminum foil to prevent the evaporation of ethyl acetate. The Ker microparticles were collected by centrifugation and then dried in a vacuum oven at room temperature until the solvent entirely evaporated. They were then observed under the scanning electron microscope (SEM). The volume and concentration to give the best morphology of the Ker microparticles were chosen for blending with the Alg solution with the same concentration. The Ker/Alg blend ratios of $3 / 7,1 / 1$, and $7 / 3$ (v/v) were prepared using the concentration of $1.6 \% \mathrm{w} / \mathrm{v}$ for each Ker and Alg solution. The mixture solution was stirred for $30 \mathrm{~min}$ to obtain homogeneous solution and then constructed into microparticles using the same method described above. The properties of the Ker/Alg blend as well as Ker and Alg microparticles were then investigated.

2.3. Characterization of Microparticles. The morphology of the microparticles was observed under the scanning electron microscope (SEM) (LEO 1450 and JEOL, JSM-6460LV, Tokyo, Japan). The microparticles were sputter coated with gold for enhancing surface conductivity. Current and voltage were adjusted to give power of $2 \mathrm{~W}(3 \mathrm{~mA}, 15 \mathrm{kV})$ for $3 \mathrm{~min}$.

The functional groups of Ker microparticles were analyzed by Fourier transform infrared (FTIR) spectroscopy (Perkin Elmer-Spectrum Gx, USA) in the spectral region of $4000-400 \mathrm{~cm}^{-1}$ at $4 \mathrm{~cm}^{-1}$ spectral resolution and 32 scans with air as reference. Before analysis, the $\mathrm{KBr}$ disk method was used for preparing samples.

The thermogravimetric analyzer (TGA) (SDTQ600, TAInstrument Co., Ltd., New Castle, DE, USA) was used for thermal stability investigation of the microparticles. Simply, $5-6 \mathrm{mg}$ of the microparticles was heated from 50 to $600^{\circ} \mathrm{C}$ with $20^{\circ} \mathrm{C} / \mathrm{min}$ of heating rate under nitrogen atmosphere.

\section{Results and Discussion}

3.1. Morphological Study. Figure 1 shows the SEM micrographs of the Ker microparticles prepared from different Ker concentrations. The results indicated that the Ker microparticles have irregular shapes including porous or hollow, spherical, oval, and bowl-like. These obtained results were similar to the microparticle shapes of other proteins such as silk fibroin (SF) prepared by the same method [14]. In $0.4 \%$ w/v concentration (Figure 1(a)), the Ker microparticles have several shapes and sizes. The shapes of the microparticle were almost hollow and bowl-like, and some debris also occurred. This debris caused by functional group of amino acid in the Ker protein reacted together and resulted to aggregate the debris. Some particles cannot close to become complete particles. In the latter case, it may be caused because of the low amount of the Ker protein used, which was not enough to enclose particles. It would be expected that the hollow or porous microparticles occurred due to high amount of water diffused out from emulsion droplets to the external phase of ethyl acetate [16, 17]. The Ker microparticles had higher uniformity in shapes when the concentration was increased to $0.8 \% \mathrm{w} / \mathrm{v}$ (Figure 1(b)). However, almost none of the microparticles had complete formation. They still have hollow and bowl-like shapes, but more dense in texture than particles prepared from lower concentration. As considering the inner wall of the microparticles, they have voids and porous structures. This void can be explained 


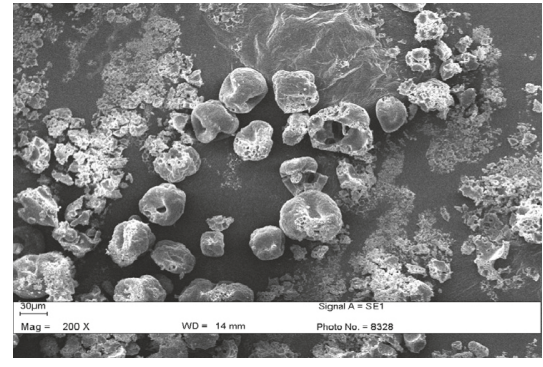

(a)

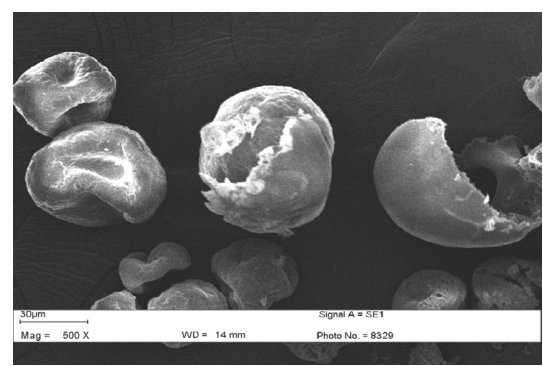

(d)

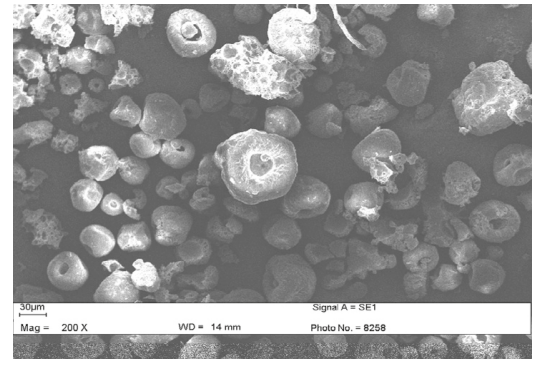

(b)

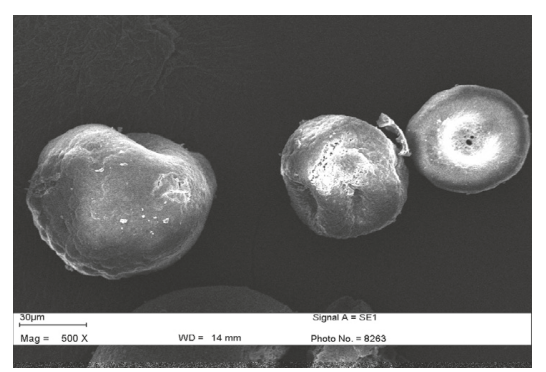

(e)

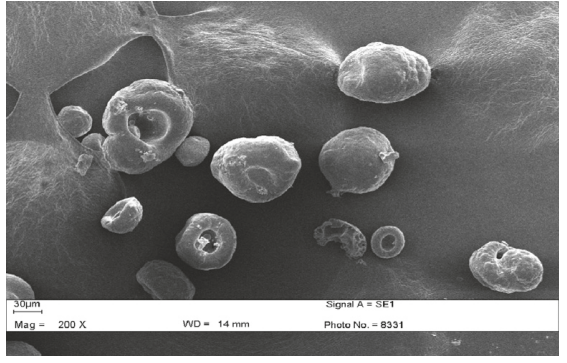

(c)

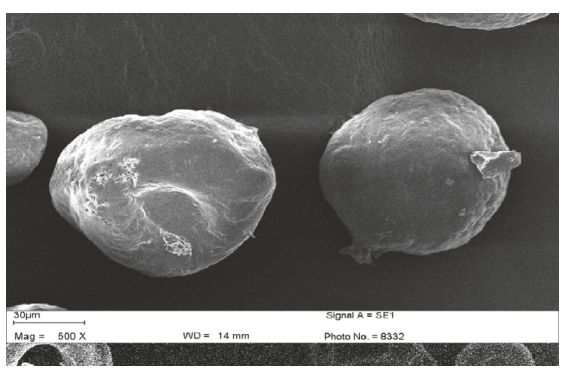

(f)

Figure 1: SEM micrographs of the Ker microparticles using $1.5 \mathrm{~mL}$ of the Ker solution with different concentrations: 0.4 (a, d), 0.8 (b, e), and $1.6(\mathrm{c}, \mathrm{f}) \% \mathrm{w} / \mathrm{v}$ under 200x (a, b, c) and 500x (d, e, f) magnifications. All bars $=30 \mu \mathrm{m}$.

by the diffusion of water into the oil phase. Moreover, the solid shell of the microparticles was formed because of the drying started from outside to inside of the droplets. Using the highest concentration of $1.6 \% \mathrm{w} / \mathrm{v}$ (Figure 1(c)), the obtained Ker microparticles still have several shapes. They have the highest dense in texture, and complete particles were constructed. Under high magnification, the particle had smooth surfaces in some areas, but rough surfaces were also observed. From the previous work, we found that the rough surface of particles can be solved by surfactant such as Span 80 [18]. The surfactant generally helped to decrease the diffusion rate of water from keratin solution and resulted in slow rate of particle formation. Figure 2 shows the Ker microparticles prepared by mixing with Span 80 . The result indicated that spherical shape microparticles with smooth surface could be obtained. However, some particles with small pores on the surfaces and voids on the inner side of particles also occurred. The several shapes of the Ker microparticles might be an advantage for many kinds of application, especially for controlled release of drugs.

$1.5 \mathrm{~mL}$ of Alg solution with a concentration of $1.6 \% \mathrm{w} / \mathrm{v}$ was prepared for the construction of Alg microparticles by using the water-in-oil (W/O) emulsification-diffusion method, as shown in Figure 3. The results indicated that the Alg microparticles were spherical with different sizes. The microparticles were separately distributed, dense texture, and rough all their surfaces. This was due to the water slowly diffused out to the oil phase. The Alg solution can move fast in the water phase to complete particle formation because of the hydrophilic functional groups in the alginate which reacted well with water.

For Ker/Alg blending microparticle construction, we had chosen the concentration of Ker solution of $1.6 \% \mathrm{w} / \mathrm{v}$ for blending with Alg and without surfactant. This was due to

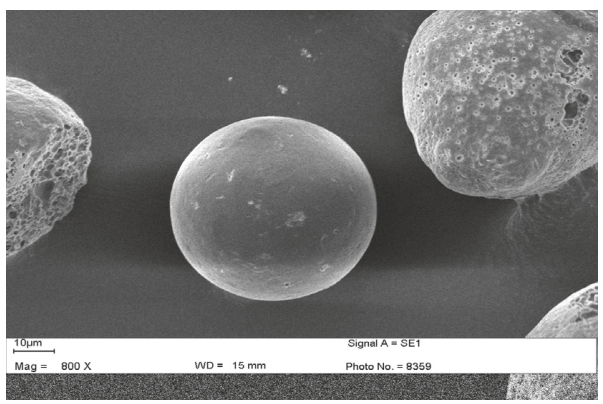

FIGURE 2: SEM micrographs of the Ker microparticles using $1.5 \mathrm{~mL}$ of the Ker solution with $1.6 \% \mathrm{w} / \mathrm{v}$ concentration under $800 \mathrm{x}$ magnification. Bars $=10 \mu \mathrm{m}$.

more hydrophilic groups in alginate which could be increased by the rheological movement of the blend solution. Figure 4 shows the microparticles of the Ker/Alg blend with a ratio of $7: 3$. The microparticles could be formed but may have several shapes and have almost been aggregated together. Considering a separated particle, it had rough cracks on its surface which were clearly observed under the highest magnification. This reveals an imbalance ratio between hydrophilic (Alg) and hydrophobic (Ker) molecules. At the Ker/Alg blend ratio of $1: 1$, the microparticles have higher spherical shapes than at the ratio of $7: 3$, as shown in Figure 5 . The results revealed that the constructed microparticles were separately distributed and were dense in texture. Considering at high magnification, the blend microparticle is miscibility. In addition, the surfaces of microparticles have about 3-5 $\mu \mathrm{m}$ in size of pores connected covering their surfaces. These pores have irregular sizes and depths. We suggested that the size and depth of pores affected by 


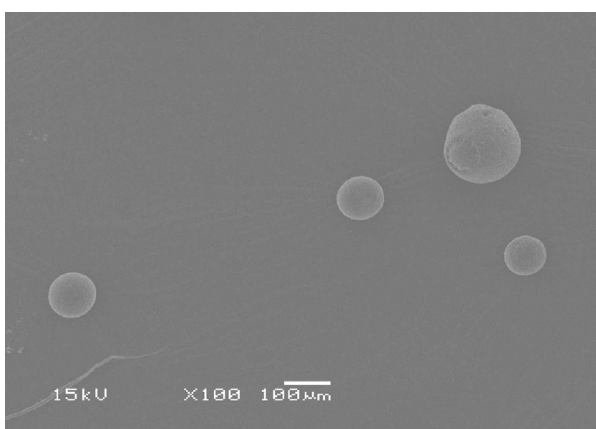

(a)

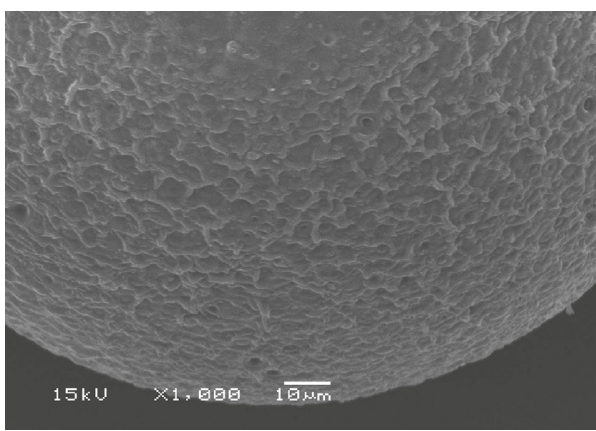

(c)

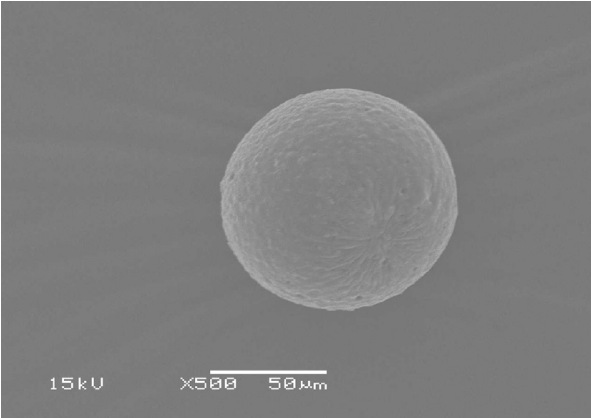

(b)

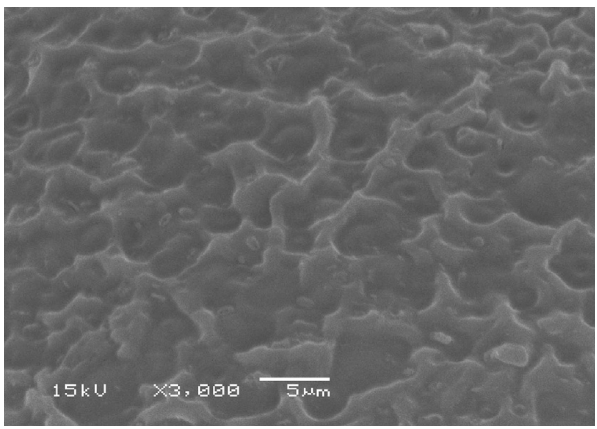

(d)

FIGURE 3: SEM micrographs of the Alg microparticles using $1.5 \mathrm{~mL}$ of the Alg solution with $1.6 \% \mathrm{w} / \mathrm{v}$ concentration under $100 \mathrm{x}$ (a), 500x (b), $1000 \mathrm{x}(\mathrm{c})$, and 3000x (d) magnifications. Bars $=100,50,10$, and $5 \mu \mathrm{m}$, respectively.

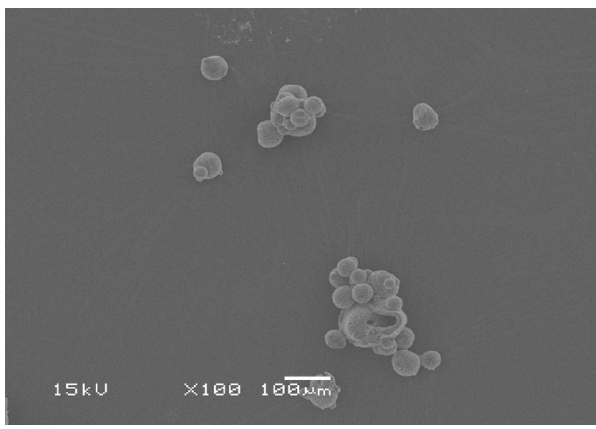

(a)

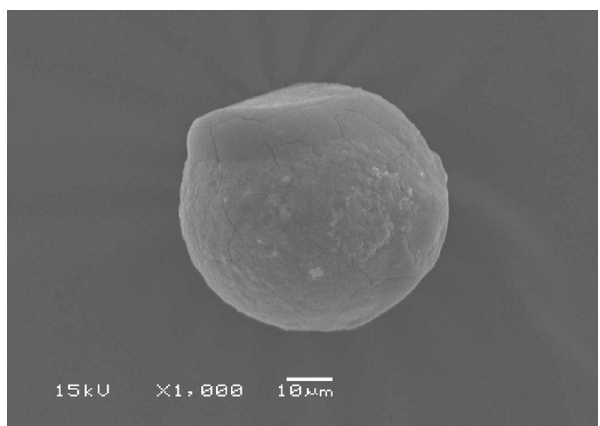

(c)

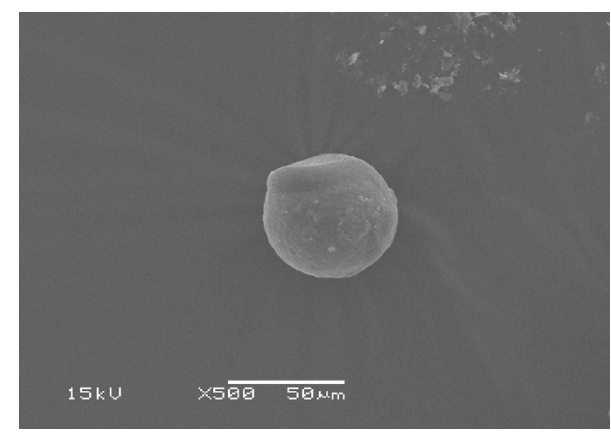

(b)

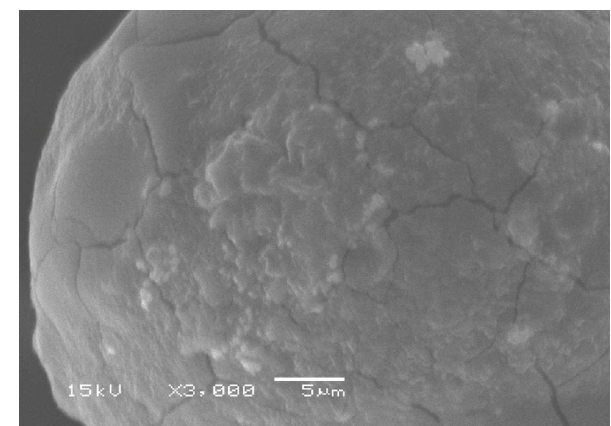

(d)

FIGURE 4: SEM micrographs of the microparticles of the Ker/Alg blend at the ratio of 7:3 under 100x (a), 500x (b), 1000x (c), and 3000x (d) magnifications. Bars $=100,50,10$, and $5 \mu \mathrm{m}$, respectively.

diffusion of alginate arranged stand between or adjacent keratin molecule. Figure 6 shows the morphology of microparticles of the Ker/Alg blend at the ratio of $3: 7$. The results indicated that the microparticles were spherical in shapes, were separately distributed, had smooth surfaces under low magnification, were dense in texture, and had 


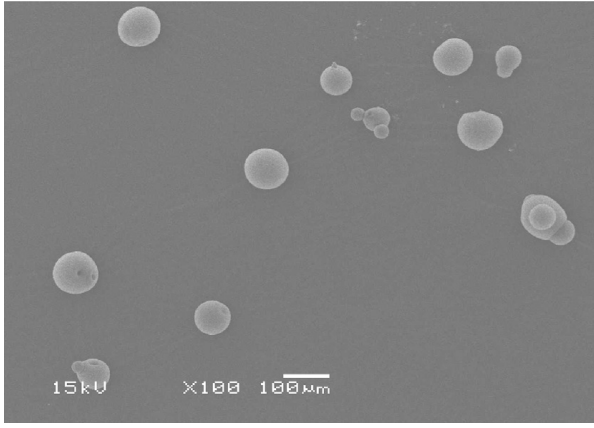

(a)

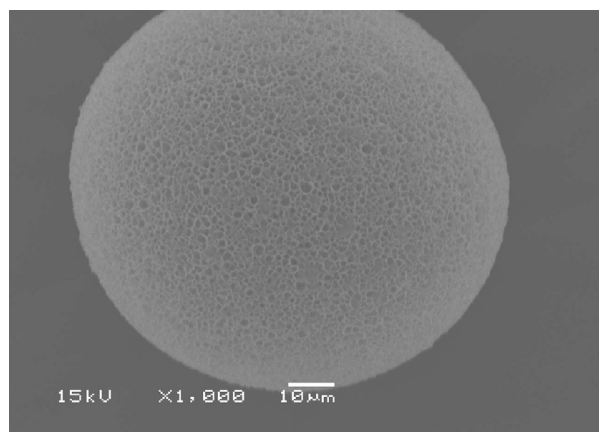

(c)

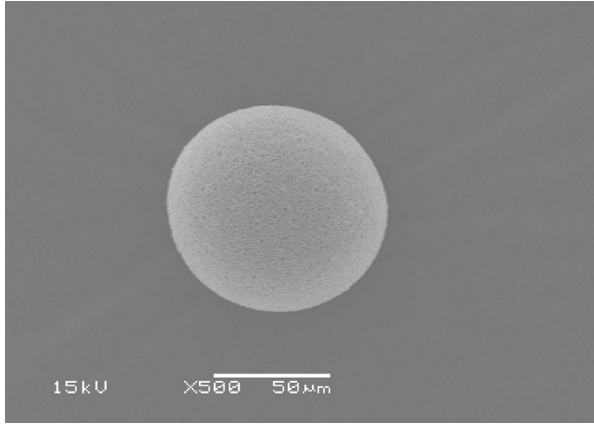

(b)

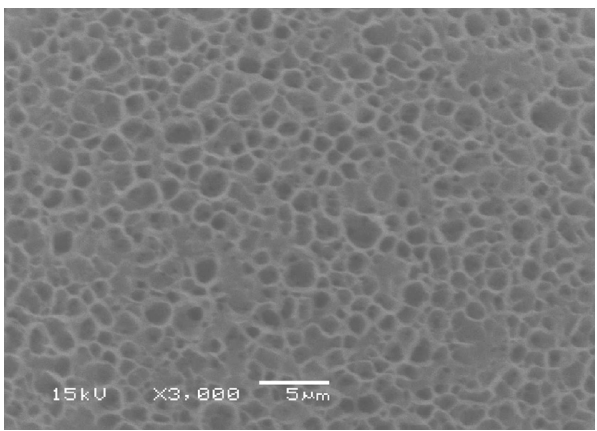

(d)

FIGURE 5: SEM micrographs of the microparticles of the Ker/Alg blend at the ratio of 1:1 under 100x (a), 500x (b), 1000x (c), and 3000x (d) magnifications. Bars $=100,50,10$, and $5 \mu \mathrm{m}$, respectively.

higher uniformity in size than other blending ratios. This indicated that the Ker and Alg molecules reacted well through functional groups which influenced the rheological profile of the blending solution as well as particle formation. Under the highest magnification, small pores $(\leq 1 \mu \mathrm{m})$ covering the surface area of the microparticles were also observed.

3.2. Secondary Structure. The secondary structure of the microparticles was investigated by the FTIR spectrophotometer, as shown in Figure 7. Generally, the peptide bond was dominant peakers of the protein which can be used to indicate Ker conformation. FTIR spectra of the Ker microparticles showed predominantly amide I at 1680 and $1645 \mathrm{~cm}^{-1}$ (random coil structure; $\mathrm{C}=\mathrm{O}$ stretching), amide II at $1524 \mathrm{~cm}^{-1}$ ( $\beta$-sheet structure; $\mathrm{N}-\mathrm{H}$ bending), and amide III at $1228 \mathrm{~cm}^{-1}$ (C-H stretching) [6]. All amide peakers were changed by blending Ker with Alg, especially at the amide III region. The Ker-blended Alg microparticles have strong $\beta$-sheet structure in both amide I and II regions. In addition, the FTIR spectra of the microparticles also displayed the peak characteristic of saccharide at $1415 \mathrm{~cm}^{-1}$. The $\beta$-sheet structure of the blended microparticles increased by increasing the ratio of $\mathrm{Alg}$, suggested that the amino groups of keratin and hydroxyl groups of alginate were interacted via $\mathrm{H}$-bond formation as well as electrostatic interaction by breaking molecular $[19,20]$. In addition, the position of amide I shifted, indicating that the hydroxyl group of alginate interacted with keratin. The Alg microparticle showed predominant broad peaks around $3399-2930 \mathrm{~cm}^{-1}$ due to $\mathrm{O}-\mathrm{H}$ and $\mathrm{C}-\mathrm{H}$ stretching and about $1038 \mathrm{~cm}^{-1}$ which is the saccharide backbone structure [21]. This revealed that the forces affected to form the Ker/Alg blend microparticles were hydrogen and negatively charged electrostatic forces [20].

3.3. Thermal Stability of Microparticles. Figure 8 shows TG thermograms of the microparticles. All of the microparticles did not completely decompose at $600^{\circ} \mathrm{C}$, with remaining weight over $20 \%$. The remaining weight at $600^{\circ} \mathrm{C}$ of blended microparticles varied as the ratio used between Ker and Alg. From the previous work, we know that the Ker microparticles exhibited at least two major weight loss phases at 265 and $289^{\circ} \mathrm{C}$ with shoulder peak at $380^{\circ} \mathrm{C}$ (data not shown). The initial weight loss at lower $100^{\circ} \mathrm{C}$ was associated with water evaporation $[22,23]$. The weight loss at $200-300^{\circ} \mathrm{C}$ were assigned as the breakdown of amino acids side chain and peptide bonds of keratin. In general, alginate had more moisture content than keratin according to their hydroxyl groups. Therefore, the Alg microparticles showed higher temperature of weight loss in the initial phase than the Ker/Alg blend. The initial weight losses of Ker/Alg blend microparticles occurred due to the moisture content of both Ker and Alg microparticles. At high Ker content (7:3, Figure 8(a)), the initial phase of weight loss showed higher temperature than other ratio, and then rapidly decreased in second phase of weight loss. Thermal decomposition in the second phase of the Alg microparticles (Figure 8(d)) started 


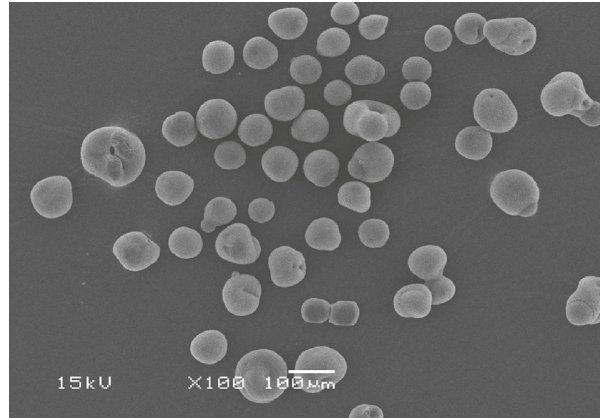

(a)

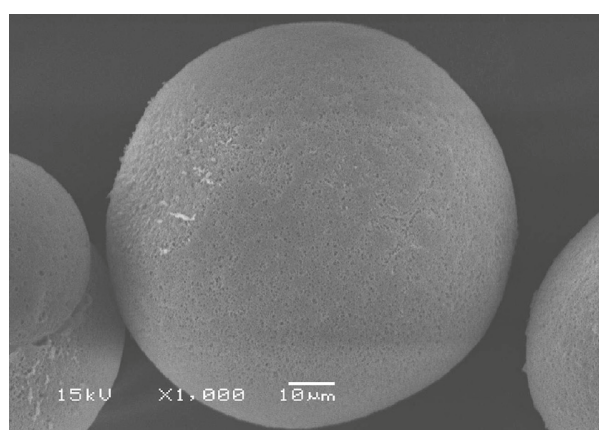

(c)

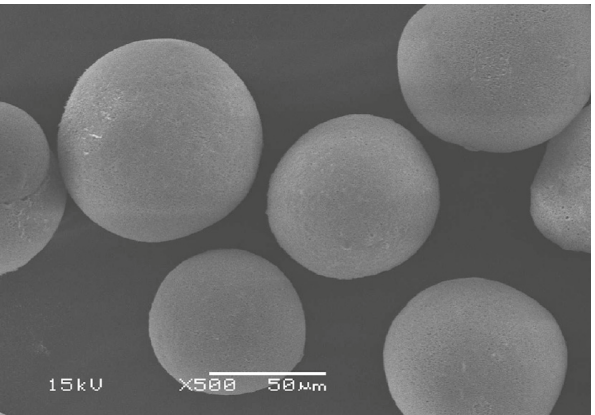

(b)

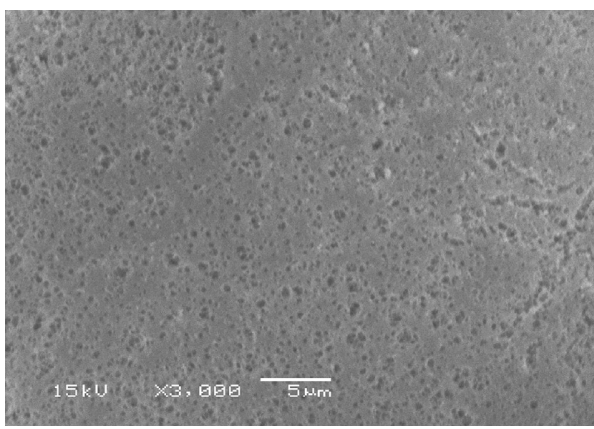

(d)

FIGURE 6: SEM micrographs of the microparticles of the Ker/Alg blend at the ratio of $3: 7$ under 100x (a), 500x (b), 1000x (c), and 3000x (d) magnifications. Bars $=100,50,10$, and $5 \mu \mathrm{m}$, respectively.

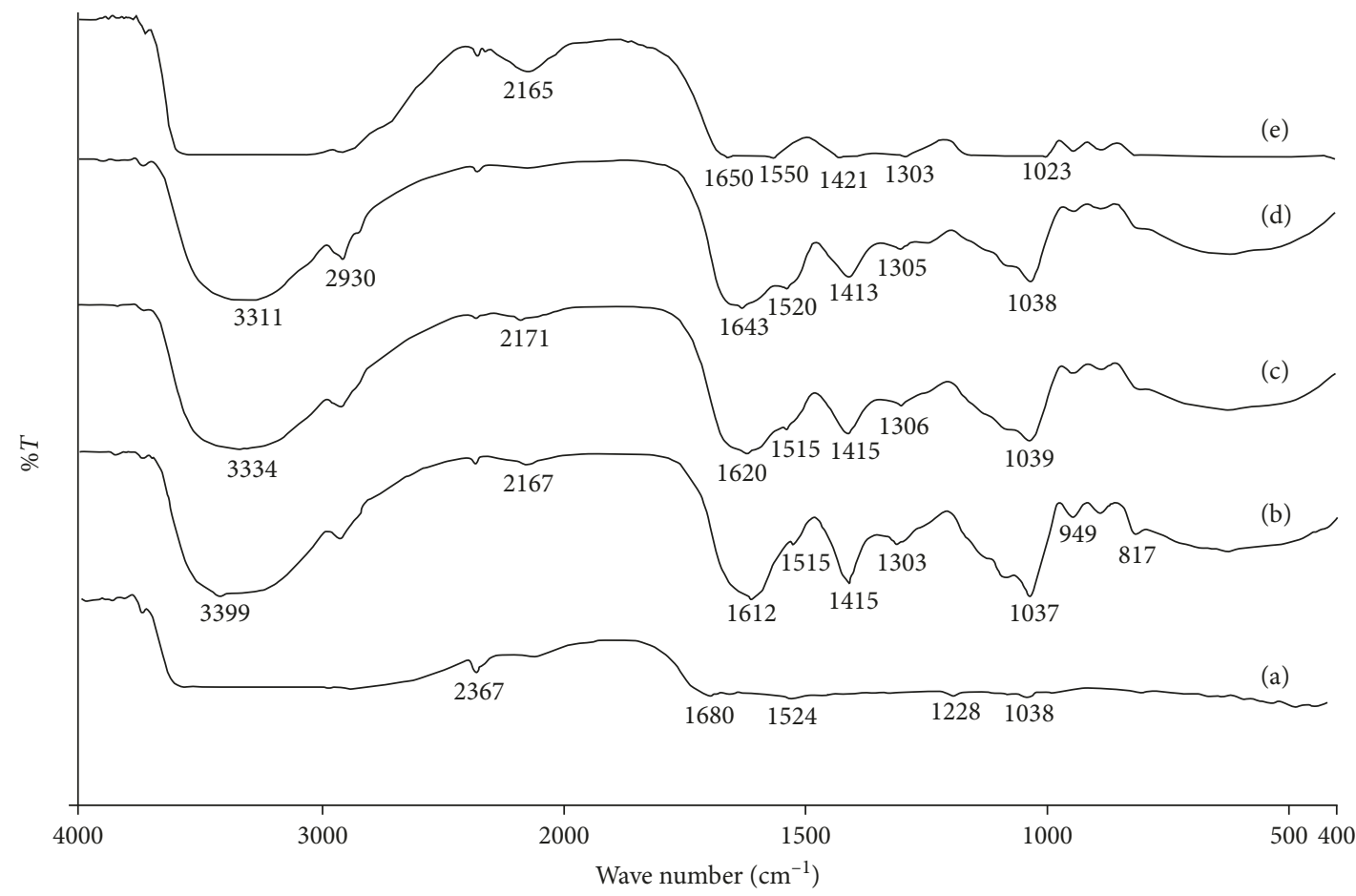

FIgURE 7: FTIR spectra of the microparticles of Ker (a) and Alg (e) and the Ker/Alg blend at ratios of 7:3 (b), 1:1 (c), and $3: 7$ (d).

at about $230^{\circ} \mathrm{C}$, and they sharply decomposed again at approximately $310^{\circ} \mathrm{C}$.

The thermal stability profile of the microparticles can be clearly revealed by differential TG (DTG) thermograms, as shown in Figure 9. The peaks around $50-60^{\circ} \mathrm{C}$ were as a result of moisture evaporation. The peaks in the range of $200-400^{\circ} \mathrm{C}$ were the decomposition temperature of Ker and Alg microparticle matrices. The temperature of maximum 


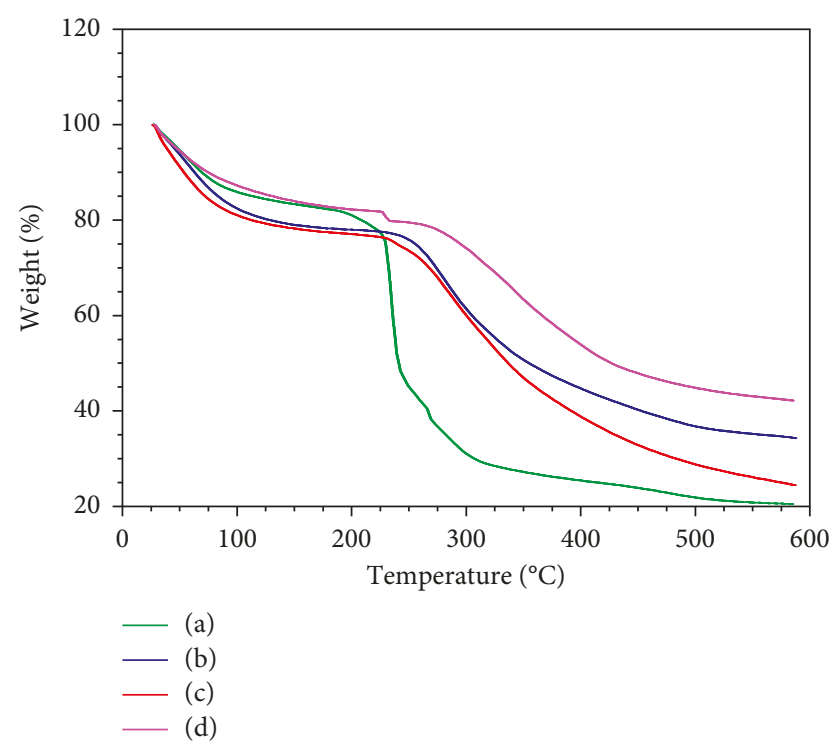

FIgURe 8: TG curves of the microparticles of Alg (d) and the Ker/Alg blend at ratios of 7:3 (a), 1:1 (c), and 3:7 (b).

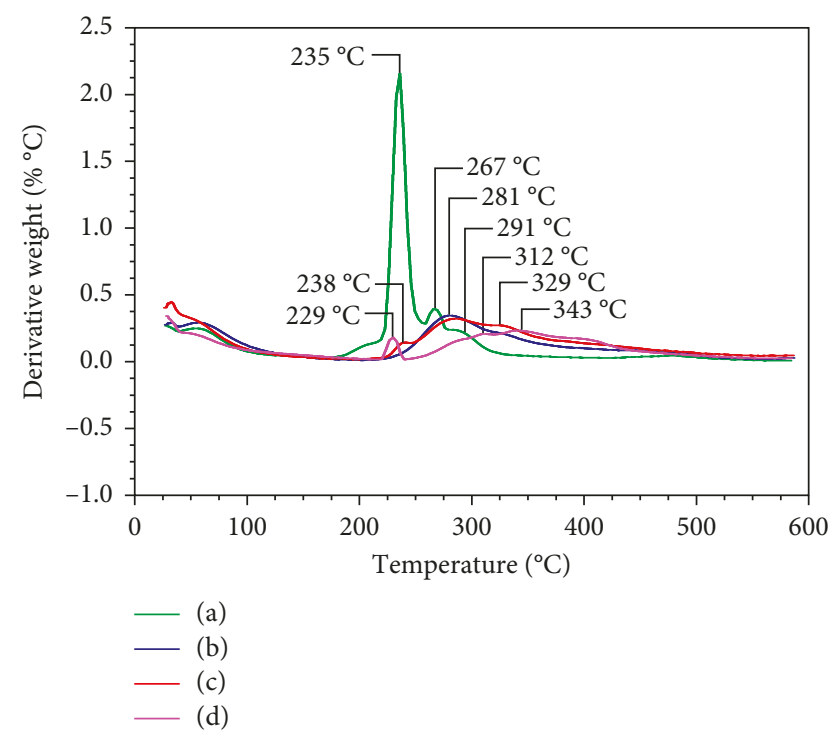

Figure 9: DTG curves of the microparticles of Alg (d) and the Ker/Alg blend at ratios of 7:3 (a), 1:1 (c), and 3:7 (b).

decomposition rate $\left(\mathrm{T}_{d, \max }\right)$ of each microparticle varied by the blend ratio. All DTG thermograms have at least two $\mathrm{T}_{d, \max }$ values. The $\mathrm{T}_{d, \max }$ values of Alg microparticles were 229 and $343^{\circ} \mathrm{C}$. This might be due to the M block and G block compositions in the chain of Alg. The mannuronic acid (M block) forms $\beta$ - $(1 \rightarrow 4)$ linkages, while the guluronic acid ( $G$ block) gives rise to $\alpha$-(1 $\rightarrow 4)$ linkages, which serves to introduce a steric hindrance around the carboxyl groups. Therefore, the G block segments provide folded and rigid structural conformations that are responsible for a pronounced stiffness of the molecular chains [11]. The blended microparticles had $\mathrm{T}_{d, \max }$ values between the Ker and Alg components, which gradually increased as the alginate in the blend ratio increased. The obtained results suggested that the interactions between Ker and Alg were formed as described by FTIR spectra.

\section{Conclusion}

A novel biopolymer-based material could be designed and constructed successfully by using the water-in-oil (W/O) emulsification-diffusion method. $1.5 \mathrm{~mL}$ and $1.6 \% \mathrm{w} / \mathrm{v}$ of $\mathrm{Ker}$ and Alg were used for microparticle construction without surfactant. With the proposed condition, the microparticles can be prepared with spherical shapes as well as different shapes and sizes. In contrast to the Ker microparticles, the Alg microparticles had spherical shape with rough surface areas. The Ker/Alg blend microparticles have different shapes, sizes, and surfaces. The conformational structure of the microparticles could be changed via the interaction between functional groups of keratin and alginate as indicated by FTIR and TGA analyses. The microparticles obtained from this work are promising for loading of both hydrophobic and hydrophilic functional molecules and delivering to the target organ via the blood circulation system.

\section{Conflicts of Interest}

The authors declare that there are no conflicts of interest regarding the publication of this paper.

\section{Acknowledgments}

This work was financially supported by Mahasarakham University in the fiscal year of 2018. The authors would like to thank the Department of Chemistry, Faculty of Science, Center of Excellence for Innovation in Chemistry, Commission on Higher Education, Ministry of Education, Thailand, for partial financial support of this work.

\section{References}

[1] P. A. Coulombe and M. B. Omary, "'Hard' and 'soft' principles defining the structure, function and regulation of keratin intermediate filaments," Current Opinion in Cell Biology, vol. 14, no. 1, pp. 110-122, 2002.

[2] B. Wang, W. Yang, J. McKittrick, and M. A. Meyers, "Keratin: structure, mechanical properties, occurrence in biological organisms, and efforts at bioinspiration," Progress in Materials Science, vol. 76, pp. 229-318, 2016.

[3] J. Wang, S. Hao, T. Luo et al., "Feather keratin hydrogel for wound repair: preparation, healing effect and biocompatibility evaluation," Colloids and Surfaces B: Biointerfaces, vol. 149, pp. 341-350, 2017.

[4] T. Luo, S. Hao, X. Chen et al., "Development and assessment of keratin nanoparticles for use as a hemostatic agent," Materials Science and Engineering: C, vol. 63, pp. 352-358, 2016.

[5] B. Ma, X. Qiao, X. Hou, and Y. Yang, "Pure keratin membrane and fibers from chicken feather," International Journal of Biological Macromolecules, vol. 89, pp. 614-621, 2016.

[6] J. Wang, S. Hao, T. Luo, Q. Yang, and B. Wang, "Development of feather keratin nanoparticles and investigation of their 
hemostatic efficacy," Materials Science and Engineering: $C$, vol. 68, pp. 768-773, 2016.

[7] V. Bertolino, G. Cavallaro, G. Lazzara et al., "Effect of the biopolymer charge and the nanoclay morphology on nanocomposite materials," Industrial \& Engineering Chemistry Research, vol. 55, no. 27, pp. 7373-7380, 2016.

[8] J. Yang, L. Liu, and S. Han, "Preparation of octyl-grafted alginate-amide gel particle and its application in Pickering emulsion," Colloids and Surfaces A: Physicochemical and Engineering Aspects, vol. 529, pp. 320-327, 2017.

[9] T. Matsumoto, M. Kawai, and T. Masuda, "Influence of concentration and mannuronate/guluronate ratio on steady flow properties of alginate aqueous systems," Biorheology, vol. 29, no. 4, pp. 411-417, 1992.

[10] G. Cavallaro, A. Gianguzza, G. Lazzara, S. Milioto, and D. Piazzese, "Alginate gel beads filled with halloysite nanotubes," Applied Clay Science, vol. 72, pp. 132-137, 2013.

[11] J.-S. Yang, Y.-J. Xie, and W. He, "Research progress on chemical modification of alginate: a review," Carbohydrate Polymers, vol. 84, no. 1, pp. 33-39, 2011.

[12] S. H. Bjørnøy, S. Mandaric, D. C. Bassett et al., "Gelling kinetics and in situ mineralization of alginate hydrogels: a correlative spatiotemporal characterization toolbox," Acta Biomaterialia, vol. 44, pp. 243-253, 2016.

[13] C. S. C. Chiew, P. E. Poh, P. Pasbakhsh, B. T. Tey, H. K. Yeoh, and E. S. Chan, "Physicochemical characterization of halloysite/alginate bionanocomposite hydrogel," Applied Clay Science, vol. 101, pp. 444-454, 2014.

[14] Y. Baimark, P. Srihanam, Y. Srisuwan, and P. Phinyocheep, "Preparation of porous silk fibroin microparticles by a waterin-oil emulsification-diffusion method," Journal of Applied Polymer Science, vol. 118, pp. 1127-1133, 2010.

[15] A. Nakamura, M. Arimoto, K. Takeuchi, and T. Fujii, “A rapid extraction procedure of human hair proteins and identification of phosphorylated species," Biological and Pharmaceutical Bulletin, vol. 25, no. 5, pp. 569-572, 2002.

[16] M. Srisa-ard and Y. Baimark, "Controlling conformational transition of silk fibroin microspheres by water vapor for controlled release drug delivery," Particulate Science and Technology, vol. 31, no. 4, pp. 379-384, 2013.

[17] O. Cheerarot and Y. Baimark, "Biodegradable silk fibroin/chitosan blend microparticles prepared by emulsification-diffusion method," e-Polymers, vol. 15, no. 2, pp. 67-74, 2015.

[18] P. Srihanam, Y. Srisuwan, T. Imsombut, and Y. Baimark, "Silk fibroin microspheres prepared by the water-in-oil emulsion solvent diffusion method for protein delivery," Korean Journal of Chemical Engineering, vol. 28, no. 1, pp. 293-297, 2011.

[19] M. Liu, Y. Zhang, C. Wu, S. Xiong, and C. Zhou, "Chitosan/halloysite nanotubes bionanocomposites: structure, mechanical properties and biocompatibility," International Journal of Biological Macromolecules, vol. 51, no. 4, pp. 566575, 2012.

[20] M. Liu, L. Dai, H. Shi, S. Xiong, and C. Zhou, "In vitro evaluation of alginate/halloysite nanotube composite scaffold," Materials Science and Engineering: C, vol. 49, pp. 700-712, 2015.

[21] Y. Baimark, M. Srisa-ard, and P. Srihanam, "Morphology and thermal stability of silk fibroin/starch blended microparticles," Express Polymer Letters, vol. 4, no. 12, pp. 781-789, 2010.

[22] C. Du, J. Jin, Y. Li, X. Kong, K. Wei, and J. Yao, "Novel silk fibroin/hydroxyapatite composite films: structure and properties," Materials Science and Engineering: C, vol. 29, no. 1, pp. 62-68, 2009.
[23] C. Mo, P. Wu, X. Chen, and Z. Shao, "The effect of water on the conformation transition of Bombyx mori silk fibroin," Vibrational Spectroscopy, vol. 51, no. 1, pp. 105-109, 2009. 


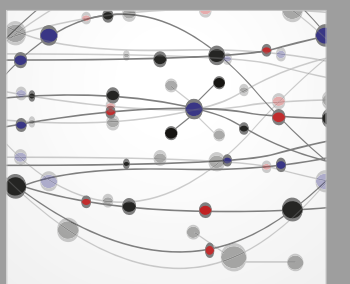

The Scientific World Journal
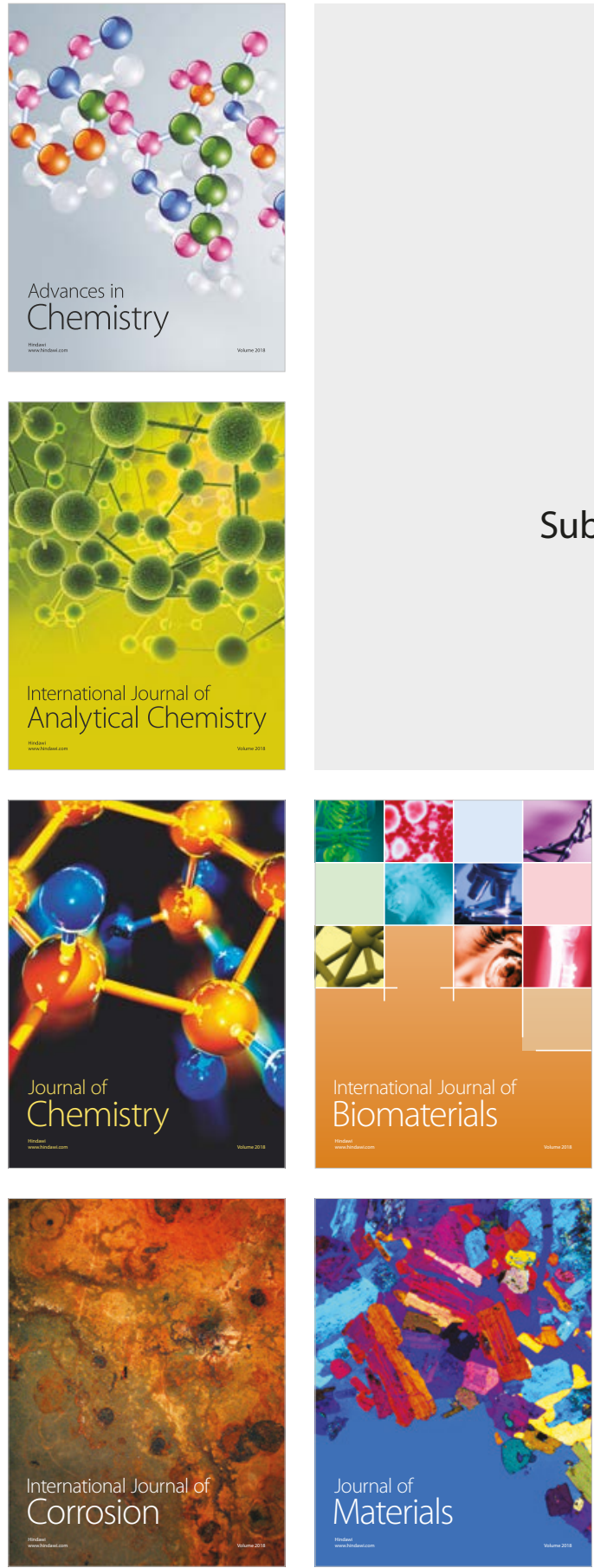

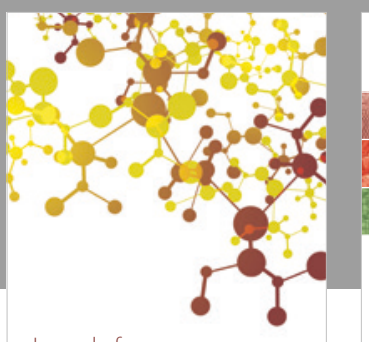

Journal of

Applied Chemistry
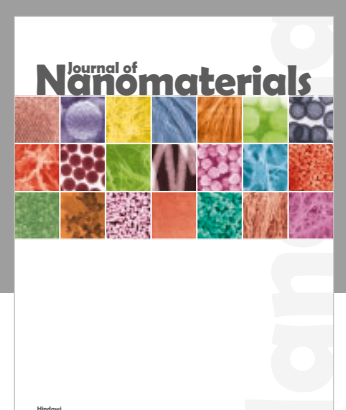

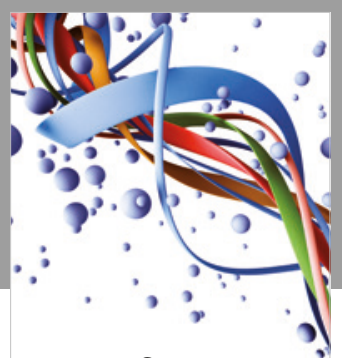

Scientifica

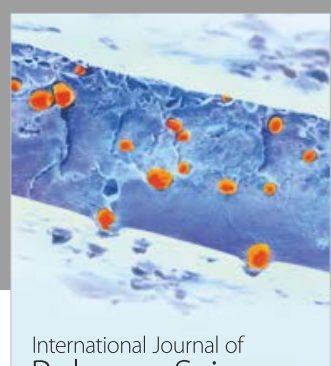

Polymer Science

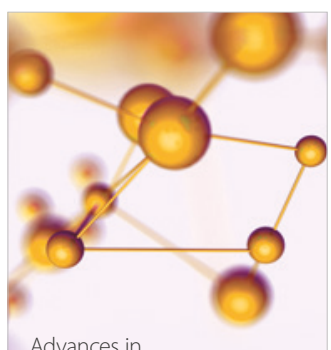

Physical Chemistry
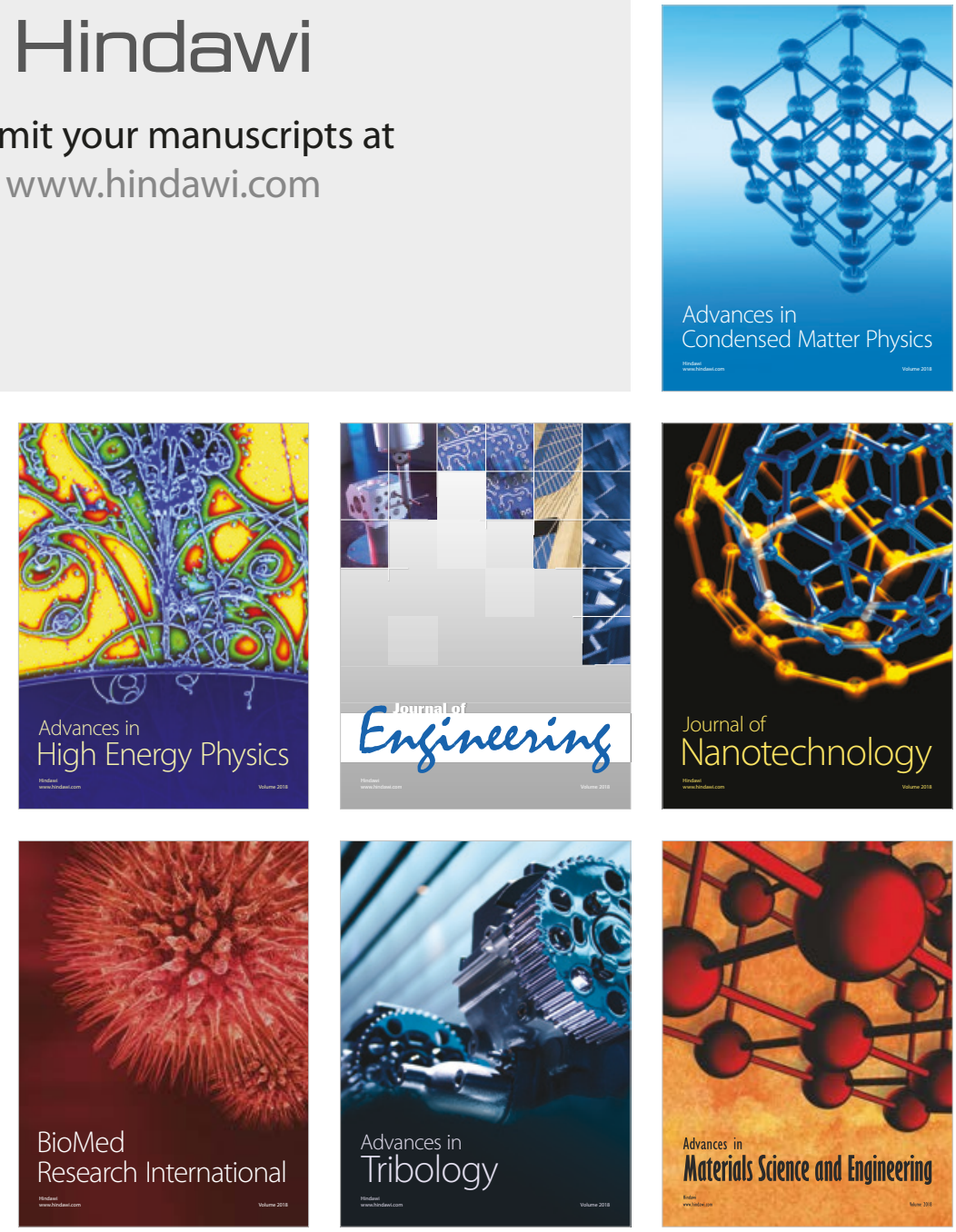